\title{
DEKOMPOSISI LEVI ALJABAR LIE AFFINE FROBENIUS $\mathfrak{a} \mathfrak{f}(2, \mathbb{R})$
}

\author{
EDI KURNIADI \\ Departemen Matematika, \\ Fakultas Matematika dan Ilmu Pengetahuan Alam, Universitas Padjadjaran, \\ Jl. Raya Bandung Sumedang KM 21, Jatinangor, Sumedang, Jawa Barat, Indonesia \\ email : edi.kurniadi@unpad.ac.id
}

\begin{abstract}
Abstrak. Dalam artikel ini dipelajari aljabar Lie affine Frobenius aff $(2, \mathbb{R})$ berdimensi 6. Aljabar Lie aff $(2, \mathbb{R})$ dapat didekomposisi menggunakan dekomposisi Levi menjadi aljabar Lie linear khusus semisederhana $\mathfrak{s l}(2, \mathbb{R})$ berdimensi 3, subaljabar Lie komutatif $\mathfrak{R} \subset \mathbb{R}^{2}$ berdimensi 2 , dan split torus $\mathfrak{T}$ berdimensi 1 sedemikian sehingga aff $(2, \mathbb{R})=$ $\mathfrak{s l}(2, \mathbb{R}) \oplus \mathfrak{R} \oplus \mathfrak{T}$. Karena aljabar Lie $\mathfrak{s l}(2, \mathbb{R})$ semisederhana maka bracket Lie-nya dapat dinyatakan sebagai $[\mathfrak{s l}(2, \mathbb{R}), \mathfrak{s l}(2, \mathbb{R})]=\mathfrak{s l}(2, \mathbb{R})$. Selanjutnya, misalkan $\mathfrak{g}=\mathfrak{R} \oplus \mathfrak{T}$ sehingga $\mathfrak{a f f}(2, \mathbb{R})=\mathfrak{s l}(2, \mathbb{R}) \oplus \mathfrak{g}$. Diperoleh bahwa $[\mathfrak{s l}(2, \mathbb{R}), \mathfrak{g}] \subseteq \mathfrak{g}$ dan $[\mathfrak{g}, \mathfrak{g}] \subseteq \mathfrak{g}$. Dalam hal ini, $\mathfrak{g}$ adalah solvable radical dari aff $(2, \mathbb{R})$.
\end{abstract}

Kata Kunci: Aljabar Lie affine, Aljabar Lie Semisederhana, Dekomposisi Levi

\section{Pendahuluan}

Aljabar Lie affine $\mathfrak{a f f}(2, \mathbb{R})$ adalah aljabar Lie non-solvable berdimensi 6 yang dapat dinyatakan dalam bentuk matriks sebagai berikut.

$$
\mathfrak{a f f}(2, \mathbb{R})=\left\{\xi(X, v)=\left(\begin{array}{cc}
X & v \\
0 & 0
\end{array}\right) ; X \in \mathfrak{g l}(2, \mathbb{R}), v \in \mathbb{R}^{2}\right\},
$$

dengan $\mathfrak{g l}(2, \mathbb{R})$ adalah aljabar Lie dari ruang vektor semua matriks berdimensi $2 \times 2$ dengan bracket Lie-nya berupa komutator matriks terhadap perkalian matriks biasa yaitu $[A, B]=A B-B A$ untuk semua $A, B \in \mathfrak{g l}(2, \mathbb{R})$. Dalam [7], aljabar Lie $\mathfrak{a f f}(2, \mathbb{R})$ adalah aljabar Lie Frobenius karena terdapat fungsional Frobenius yang mengakibatkan stabilizer $\mathfrak{a f f}(2, \mathbb{R})$ sama dengan himpunan vektor nol.

Sifat lain yang dimiliki oleh aljabar Lie affine $\mathfrak{a f f}(2, \mathbb{R})$ adalah ketidak semisederhanaannya. Dengan mengonstruksi Killing form-nya, dapat ditunjukkan bahwa matriks representasinya mempunyai determinan sama dengan nol atau dengan kata lain, Killing form dari aff $(2, \mathbb{R})$ adalah degenerate. Ditinjau dari sifat turunannya, semua turunan $\mathfrak{a f f}(2, \mathbb{R})$ adalah inner [3]. Berbeda dengan sifat $\mathfrak{a f f}(2, \mathbb{R})$ yang tidak semisederhana, dalam penelitian ini dibuktikan bahwa hasil dekomposisi $\mathfrak{a f f}(2, \mathbb{R})$ 
memuat aljabar Lie semisederhana $\mathfrak{s l}(2, \mathbb{R})$ yaitu aljabar Lie dari ruang vektor matriks berdimensi $2 \times 2$ dengan trace sama dengan nol. Meskipun hasil ini sudah diperoleh oleh [7], tetapi konstruksi yang dilakukan berbeda dengan hasil sebelumnya.

Dalam [4] telah dikonstruksi basis untuk aff $(2, \mathbb{R})$ sebagai berikut.

$$
S=\left\{x_{1}=\left(\begin{array}{lll}
1 & 0 & 0 \\
0 & 0 & 0 \\
0 & 0 & 0
\end{array}\right), \cdots, x_{6}=\left(\begin{array}{lll}
0 & 0 & 0 \\
0 & 0 & 1 \\
0 & 0 & 0
\end{array}\right)\right\} .
$$

Vektor-vektor basis $\mathfrak{g l}(2, \mathbb{R})$ yang dimuat di $\mathfrak{s l}(2, \mathbb{R})$ dapat dinyatakan sebagai berikut.

$$
\overrightarrow{x_{2}}=\left(\begin{array}{ll}
0 & 1 \\
0 & 0
\end{array}\right), \overrightarrow{x_{3}}=\left(\begin{array}{ll}
0 & 0 \\
1 & 0
\end{array}\right) .
$$

Oleh karena itu perlu dikonstruksi suatu vektor $\theta \in \mathfrak{s l}(2, \mathbb{R})$ sedemikian sehingga $S=\left\{\overrightarrow{x_{2}}, \overrightarrow{x_{3}}, \theta\right\}$ bisa menjadi basis untuk $\mathfrak{s l}(2, \mathbb{R})$.

Tujuan penelitian ini adalah untuk mendekomposisi aljabar Lie affine $\mathfrak{a f f}(2, \mathbb{R})$ menggunakan dekomposisi Levi dalam tiga komponen, yaitu aljabar Lie semisederhana $\mathfrak{s l}(2, \mathbb{R})$ berdimensi 3 , subaljabar komutatif $\mathfrak{R}$ berdimensi 2 , dan split torus $\mathfrak{T}$ berdimensi 1. Lebih jauh, telah diperoleh bahwa $\theta=\overrightarrow{x_{1}}-\overrightarrow{x_{4}}=\left(\begin{array}{cc}1 & 0 \\ 0 & -1\end{array}\right)$, dengan $\overrightarrow{x_{1}}=\left(\begin{array}{ll}1 & 0 \\ 0 & 0\end{array}\right)$ dan $\overrightarrow{x_{4}}=\left(\begin{array}{ll}0 & 0 \\ 0 & 1\end{array}\right)$. Jelas bahwa $\theta$ termuat di $\mathfrak{s l}(2, \mathbb{R})$. Dengan kata lain, $\mathfrak{s l}(2, \mathbb{R})=\left\langle x_{2}, x_{3}, \theta\right\rangle$. Di sisi lain, diperoleh juga subaljabar Lie komutatif yang dibangun oleh dua vektor $\mathfrak{R}=\left\langle x_{5}, x_{6}\right\rangle$, dan split torus yang dibangun oleh satu vektor $\mathfrak{T}=\langle t\rangle$, dengan $t=\operatorname{diag}\{0,0,0,1,1\}[7]$.

Artikel ini ditulis dengan menggunakan sistematika penulisan sebagai berikut. Bagian pertama adalah pendahuluan yang menjelaskan tentang kajian yang telah dilakukan oleh peneliti sebelumnya, motivasi dan latar belakang penelitian, dan signifikansi penelitian. Bagian kedua menjelaskan landasan teori yang digunakan dalam membahas hasil utama dalam penelitian ini seperti aljabar Lie affine, aljabar Lie semisederhana, dan dekomposisi Levi. Bagian ketiga adalah pembahasan, yaitu pembuktian bahwa aljabar Lie affine $\mathfrak{a f f}(2, \mathbb{R})$ dapat dinyatakan dalam dekomposisi Levi $\mathfrak{a f f}(2, \mathbb{R})=\mathfrak{s l}(2, \mathbb{R}) \oplus \mathfrak{R} \oplus \mathfrak{T}$.

\section{Landasan Teori}

Dalam bagian ini akan dibahas kembali beberapa materi yang berkaitan dengan penelitian ini, seperti aljabar Lie, aljabar Lie Frobenius, aljabar Lie semisederhana, dan dekomposisi Levi.

Definisi 2.1. [5] Ruang vektor real $\mathfrak{g}$ disebut aljabar Lie jika ruang vektor $\mathfrak{g}$ tersebut dilengkapi dengan pemetaan bilinear

$$
[,]: \mathfrak{g} \ni(a, b) \mapsto[a, b] \in \mathfrak{g},
$$

di mana untuk setiap $a, b, c \in \mathfrak{g}$ berlaku $[a, b]=[b, a]$ dan

$$
[a,[b, c]]+[b,[c, a]]+[c,[a, b]]=0 .
$$


Pemetaan bilinear [,] yang memenuhi kedua sifat tersebut dinamakan bracket Lie dan persamaan (2.2) dinamakan identitas Jacobi. Contoh aljabar Lie adalah ruang vektor real $\mathbb{R}$ dengan bracket Lie $[a, b]=0$ untuk setiap $a, b \in \mathbb{R}$. Selain itu, ruang vektor $\mathbb{R}^{3}$ dengan bracket Lie-nya berupa hasil kali silang juga merupakan contoh aljabar Lie.

Untuk aljabar Lie $\mathfrak{g}$, bracket $[\mathfrak{g}, \mathfrak{g}]$ didefinisikan sebagai berikut [5].

$$
[\mathfrak{g}, \mathfrak{g}]=\{[x, y] ; x, y \in \mathfrak{g}\}
$$

Misalkan $\mathfrak{g}^{*}$ adalah ruang dual dari $\mathfrak{g}$ yang didefinisikan sebagai berikut.

$$
\mathfrak{g}^{*}=\{f ; f: \mathfrak{g} \rightarrow \mathbb{R} \text { suatu pemetaan linear }\}
$$

Stabilizer dari $\mathfrak{g}$ di titik $f_{0} \in \mathfrak{g}^{*}$ didefinisikan oleh

$$
\mathfrak{g}_{f_{0}}=\left\{x \in \mathfrak{g} ; f_{0}([x, y])=0, \forall y \in \mathfrak{g}\right\} .
$$

Stabilizer sangat erat kaitannya dalam menentukan ke-Frobenius-an suatu aljabar Lie. Lebih jauh akan disampaikan lebih detail dalam pembahasan selanjutnya.

Selanjutnya akan dibahas tentang kriteria suatu aljabar Lie agar dikatakan semisederhana, yang secara formal dinyatakan dalam definisi berikut.

Definisi 2.2. [2] Aljabar Lie $\mathfrak{g}$ dikatakan semisederhana jika $\mathfrak{g}$ tidak mempunyai ideal solvable lain kecuali $\{0\}$.

Sebelum memberikan kriteria lain tentang kesemisederhanaan, diberikan definisi Killing form sebagai berikut.

Definisi 2.3. [5] Misalkan $\mathfrak{g}$ aljabar Lie yang dilengkapi bilinear form

$$
\mathfrak{K}: \mathfrak{g} \times \mathfrak{g} \rightarrow \mathbb{R},
$$

yang didefinisikan oleh $\mathfrak{K}(\alpha, \beta)=\operatorname{Tr}(\operatorname{ad}(\alpha)$ ad $(\beta))$ untuk setiap $\alpha, \beta \in \mathfrak{g}$. Bilinear form $\mathfrak{K}$ dikatakan Killing form jika $\mathfrak{K}$ simetrik.

Suatu Killing form $\mathfrak{K}$ dikatakan non-degenerate jika determinan matriks representasi $\mathfrak{K}$ tidak sama dengan nol. Notasi tersebut membawa kepada kriteria kesemisederhanaan sebagai berikut.

Teorema 2.4. [5] Misalkan $\mathfrak{g}$ aljabar Lie dengan Killing form $\mathfrak{K}$. Jika $\mathfrak{K}$ nondegenerate maka $\mathfrak{g}$ semisederhana.

Karena aljabar Lie $\mathfrak{g}$ dalam penelitian ini atas lapangan real $\mathbb{R}$ maka konvers pernyataan dalam Teorema 2.4 berlaku. Dengan kata lain, jika $\mathfrak{g}$ aljabar semisederhana real maka Killing form-nya adalah non-degenerate.

Misalkan $\mathfrak{g l}(2, \mathbb{R})$ adalah aljabar Lie dari ruang vektor matriks $2 \times 2$ terhadap bracket Lie komutator matriks. Aljabar Lie $\mathfrak{s l}(2, \mathbb{R})=\{A \in \mathfrak{g l}(2, \mathbb{R}) ; \operatorname{Tr}(A)=0\}=$ $\left\langle x_{1}, x_{2}, x_{3}\right\rangle$ dengan bracket Lie

$$
\begin{aligned}
& {\left[x_{1}, x_{2}\right]=2 x_{2},} \\
& {\left[x_{1}, x_{3}\right]=-2 x_{3},} \\
& {\left[x_{2}, x_{3}\right]=x_{1}}
\end{aligned}
$$


adalah contoh aljabar Lie semisederhana. Killing form-nya non-degenerate karena determinan matriks representasinya tidak sama dengan nol. Selanjutnya, aljabar Lie Heisenberg $\mathcal{H}_{3}=\langle x, y, z\rangle$ dengan bracket Lie

$$
\left[x_{1}, x_{2}\right]=x_{2}
$$

adalah aljabar Lie yang tidak semisederhana karena Killing form-nya degenerate, atau matriks representasinya mempunyai determinan sama dengan nol.

Misalkan didefinisikan barisan aljabar Lie $\mathfrak{g}^{(k)}$ dengan $k \geq 1$ sebagai berikut.

$$
\mathfrak{g}^{(1)}=\mathfrak{g}, \mathfrak{g}^{(2)}=\left[\mathfrak{g}^{(1)}, \mathfrak{g}^{(1)}\right], \cdots, \mathfrak{g}^{(k)}=\left[\mathfrak{g}^{(k-1)}, \mathfrak{g}^{(k-1)}\right] \cdots
$$

Aljabar Lie $\mathfrak{g}$ dikatakan solvable jika terdapat $n>0$ sedemikian sehingga $\mathfrak{g}^{(n)}=\{0\}$ dan $\mathfrak{g}^{(n-1)} \neq\{0\}$. Suatu aljabar Lie $\mathfrak{g}$ hingga senantiasa memuat ideal solvable maksimal, yang merupakan penjumlahan semua ideal-ideal solvable di $\mathfrak{g}$, dan ideal tersebut dinamakan radical solvable, dinotasikan $\mathcal{S R}(\mathfrak{g})$. Khususnya untuk aljabar Lie real $\mathfrak{g}$ hingga, diperoleh

$$
\mathcal{S R}(\mathfrak{g})=\{\alpha \in \mathfrak{g} ; \operatorname{Tr}(\operatorname{ad}(\alpha) \operatorname{ad}(\beta))=0, \forall \beta \in[\mathfrak{g}, \mathfrak{g}]\} .
$$

Definisi 2.5. [8] Aljabar Lie $\mathfrak{g}$ dikatakan Frobenius jika stabilizer $\mathfrak{g}_{f_{0}}$ untuk suatu $f_{0} \in \mathfrak{g}^{*}$ sama dengan $\{0\}$.

Lebih jauh, kita definisikan barisan aljabar Lie $\mathfrak{g}^{k}$ dengan $k \geq 1$ sebagai berikut.

$$
\mathfrak{g}^{1}=\mathfrak{g}, \mathfrak{g}^{2}=[\mathfrak{g}, \mathfrak{g}], \cdots, \mathfrak{g}^{k}=\left[\mathfrak{g}^{k-1}, \mathfrak{g}\right], \cdots
$$

Aljabar Lie $\mathfrak{g}$ dikatakan nilpoten jika terdapat $n>0$ sedemikian sehingga $\mathfrak{g}^{n}=\{0\}$ dan $\mathfrak{g}^{n-1} \neq\{0\}$. Perhatikan bahwa $\mathfrak{g}^{(k)} \subset \mathfrak{g}^{k}$. Oleh karena itu, setiap aljabar Lie nilpoten adalah aljabar Lie solvable.

Teorema 2.6. [5] Misalkan $\mathfrak{g}$ Aljabar Lie real berdimensi hingga. Jika $\mathfrak{g}$ tidak solvable maka terdapat sub-aljabar semisederhana $\mathcal{S}$ dari $\mathfrak{g}$ sedemikian sehingga

$$
\mathfrak{g}=\mathcal{S} \oplus \mathcal{S R}(\mathfrak{g})
$$

Dalam hal ini, $S \simeq \mathfrak{g} / \mathcal{S R}(\mathfrak{g})$ dan berlaku

$$
[\mathcal{S}, \mathcal{S}]=\mathcal{S},[\mathcal{S}, \mathcal{S R}(\mathfrak{g})] \subseteq \mathcal{S R}(\mathfrak{g}),[\mathcal{S R}(\mathfrak{g}), \mathcal{S R}(\mathfrak{g})] \subseteq \mathcal{S R}(\mathfrak{g})
$$

\section{Pembahasan}

Sebelum membahas hasil utama dalam penelitian ini, terlebih dahulu kita bahas beberapa sifat aljabar Lie affine $\mathfrak{a f f}(2, \mathbb{R})$ sebagai berikut. Menggunakan basis pada (1.2), diperoleh bracket Lie untuk aljabar Lie affine $\mathfrak{a f f}(2, \mathbb{R})$ sebagai berikut.

$$
\begin{array}{r}
{\left[x_{1}, x_{2}\right]=x_{2},\left[x_{1}, x_{3}\right]=-x_{3},\left[x_{1}, x_{5}\right]=x_{5},} \\
{\left[x_{2}, x_{3}\right]=x_{1}-x_{4},\left[x_{2}, x_{4}\right]=x_{2},\left[x_{2}, x_{6}\right]=x_{5},} \\
{\left[x_{3}, x_{4}\right]=-x_{3},\left[x_{3}, x_{5}\right]=x_{6}, \quad\left[x_{4}, x_{6}\right]=x_{6} .}
\end{array}
$$

Proposisi 3.1. [4] Aljabar Lie affine $\mathfrak{a f f}(2, \mathbb{R})$ adalah aljabar Lie Frobenius. 
Bukti. Pertama dipilih fungsional linear $f_{0}=x_{2}^{*}+x_{6}^{*} \in \mathfrak{a f f}(2, \mathbb{R})$. Kemudian untuk $x_{k} \in \mathfrak{a f f}(2, \mathbb{R})$ didefinisikan $\left(x_{2}^{*}+x_{6}^{*}\right)\left(x_{k}\right)=1,(k=1,2, \cdots, 6)$ jika $k=2$ atau $k=6$ dan nol untuk selainnya. Perhatikan bahwa hasil bracket Lie $\left[x_{i}, x_{j}\right]$ untuk setiap $1 \leq i, j \leq 6$ senantiasa memuat $x_{2}$ atau $x_{6}$. Dengan kata lain, $f_{0}\left(\left[x_{i}, x_{j}\right]\right)$ tidak semuanya nol. Oleh karena itu, $x_{k} \notin \mathfrak{g}_{f_{0}}$ untuk setiap $k=1,2, \cdots, 6$. Jadi, $\mathfrak{g}_{f_{0}}=\{0\}$. Hal ini mengakibatkan $\mathfrak{a} \mathfrak{f}(2, \mathbb{R})$ adalah aljabar Lie Frobenius.

Proposisi 3.2. Aljabar Lie $\mathfrak{a f f}(2, \mathbb{R})$ tidak semisederhana.

Bukti. Kita gunakan kontraposisi pernyataan berikut: Jika $\mathfrak{g}$ aljabar Lie semisederhana maka $[\mathfrak{g}, \mathfrak{g}]=\mathfrak{g}$. Perhatikan bahwa berdasarkan perhitungan bracket Lie pada persamaan (3.1) diperoleh bahwa

$$
[\mathfrak{a f f}(2, \mathbb{R}), \mathfrak{a f f}(2, \mathbb{R})]=\left\langle x_{1} x_{4}, x_{2}, x_{3}, x_{5}, x_{6}\right\rangle \neq \mathfrak{a f f}(2, \mathbb{R}) .
$$

Dengan demikian, aljabar Lie $\mathfrak{a f f}(2, \mathbb{R})$ tidak semisederhana.

Meskipun $\mathfrak{a f f}(2, \mathbb{R})$ tidak semisederhana tetapi semua turunan $\mathfrak{a f f}(2, \mathbb{R})$ adalah turunan dalam atau inner derivation.

Proposisi 3.3. Aljabar Lie aff $(2, \mathbb{R})$ non-solvable.

Bukti. Perhatikan bahwa

$$
\begin{aligned}
\mathfrak{a f f}(2, \mathbb{R})^{(2)} & =[\mathfrak{a f f}(2, \mathbb{R}), \mathfrak{a f f}(2, \mathbb{R})], \\
& =\left\langle x_{1}-x_{4}, x_{2}, x_{3}, x_{5}, x_{6}\right\rangle .
\end{aligned}
$$

Selanjutnya, dengan menghitung $\mathfrak{a f f}(2, \mathbb{R})^{(3)}$ juga diperoleh

$$
\begin{aligned}
\mathfrak{a} \mathfrak{f f}(2, \mathbb{R})^{(3)} & =\left[\mathfrak{a} \mathfrak{f} \mathfrak{f}(2, \mathbb{R})^{(2)}, \mathfrak{a} \mathfrak{f} \mathfrak{f}(2, \mathbb{R})^{(2)}\right], \\
& =\left\langle x_{1}-x_{4}, x_{2}, x_{3}, x_{5}, x_{6}\right\rangle .
\end{aligned}
$$

Oleh karena itu, tidak terdapat $n>0$ yang mengakibatkan $\mathfrak{a f f}(2, \mathbb{R})^{(n)}=0$. Jadi $\mathfrak{a f f}(2, \mathbb{R})$ adalah aljabar Lie non-solvable. Akibatnya, aljabar Lie affine $\mathfrak{a f f}(2, \mathbb{R})$ juga tidak nilpoten.

Proposisi 3.4. Untuk aljabar Lie affine Frobenius non-solvable aff $(2, \mathbb{R})$ terdapat aljabar Lie semisederhana $\mathfrak{s l}(2, \mathbb{R})$ dan $\mathfrak{g}=\mathfrak{R} \oplus \mathfrak{T}$ dengan $\mathfrak{R}$ subaljabar Lie komutatif dan $\mathfrak{T}$ split torus sedemikian sehingga

$$
\mathfrak{a f f}(2, \mathbb{R})=\mathfrak{s l}(2, \mathbb{R}) \oplus \mathfrak{g} .
$$

Bukti. Kita gunakan basis dalam persamaan (1.2) dengan bracket Lie-nya diberikan oleh persamaan (3.1). Produk Bracket Lie $[\mathfrak{a f f}(2, \mathbb{R}), \mathfrak{a f f}(2, \mathbb{R})]$ dibangun oleh vektor-vektor $S=\left\{x_{1}-x_{4}, x_{2}, x_{3}, x_{5}, x_{6}\right\}$. Perhatikan bahwa jika kita memisalkan $\theta:=x_{1}-x_{4}$ dan menghitung ulang bracket pada subaljabar Lie $\mathfrak{P}$ yang dibangun oleh $S=\left\{\theta, x_{2}, x_{3}\right\}$, maka kita peroleh

$$
\begin{aligned}
{\left[\theta, x_{2}\right] } & =2 x_{2}, \\
{\left[\theta, x_{3}\right] } & =-2 x_{3}, \\
{\left[x_{2}, x_{3}\right] } & =\theta .
\end{aligned}
$$


Dengan menghitung langsung matriks representasi Killing form

$$
\mathfrak{K}: \mathfrak{P} \times \mathfrak{P} \ni(x, y) \mapsto \mathfrak{K}(x, y)=\operatorname{Tr}(\operatorname{ad}(x) \operatorname{ad}(y)),
$$

didapat $A_{\mathfrak{K}}=\left(\begin{array}{lll}0 & 0 & 4 \\ 0 & 8 & 0 \\ 4 & 0 & 0\end{array}\right)$, dimana determinan $A_{\mathfrak{K}}=-128 \neq 0$ (Lihat [6]). Jadi, $\mathfrak{P}$ semisederhana. Dengan kata lain, $\mathfrak{P}$ adalah $\mathfrak{s l}(2, \mathbb{R})$.

Selanjutnya dari himpunan $S$ di atas, kita bisa memilih subaljabar $\mathfrak{R}$ berdimensi dua yang dibangun oleh $S^{\prime \prime}=\left\{x_{5}, x_{5}\right\}$. Karena $\left[x_{5}, x_{6}\right]=0$ maka $\mathfrak{R}$ komutatif. Selanjutnya kita perlu mencari split torus $\mathfrak{T}$, yaitu aljabar Lie komutatif yang memuat turunan diagonalizable dari $\mathfrak{s l}(2, \mathbb{R}) \oplus \mathfrak{R}$. Konstruksi $\mathfrak{T}$ yang lebih detail bisa dilihat dalam [1]. Lebih jauh diperoleh $\mathfrak{T}=\langle t\rangle$ dengan $t=\operatorname{diag}(0,0,0,1,1)$. Dalam hal ini, $\mathcal{S R}(\mathfrak{a f f}(2, \mathbb{R})=\mathfrak{g}$ dan

$$
\mathfrak{s l}(2, \mathbb{R}) \simeq \mathfrak{a f f}(2, \mathbb{R}) / \mathcal{S R}(\mathfrak{a f f}(2, \mathbb{R})) .
$$

Selanjutnya, diperoleh bahwa

$$
\begin{aligned}
{[\mathfrak{s l}(2, \mathbb{R}), \mathfrak{s l}(2, \mathbb{R})] } & =\mathfrak{s l}(2, \mathbb{R}), \\
{[\mathfrak{s l}(2, \mathbb{R}), \mathfrak{g}] } & \subseteq \mathfrak{g} \\
{[\mathfrak{g}, \mathfrak{g}] } & \subseteq \mathfrak{g} .
\end{aligned}
$$

\section{Kesimpulan}

Telah dibuktikan bahwa aljabar Lie affine $\mathfrak{a f f}(2, \mathbb{R})$ dapat didekomposisi dalam aljabar Lie semisederhana $\mathfrak{s l}(2, \mathbb{R})$ dan solvable radical $\mathcal{S} \mathcal{R}(\mathfrak{a f f}(2, \mathbb{R})$. Dekomposisi tersebut dinamakan dekomposisi Levi yaitu :

$$
\mathfrak{a f f}(2, \mathbb{R})=\mathfrak{s l}(2, \mathbb{R}) \oplus \mathcal{S} \mathcal{R}(\mathfrak{a f f}(2, \mathbb{R})) .
$$

\section{Ucapan Terima Kasih}

Penulis mengucapkan terima kasih kepada Universitas Padjadjaran yang telah mendanai penelitian ini melalui Riset Percepatan Lektor Kepala (RPLK) tahun 2021 dengan nomor kontrak 1959/UN6.3.1/PT.00/2021.

\section{Daftar Pustaka}

[1] Ayala, V., Kizil, E., and Tribuzy, I. D. A., 2012, On an algorithm for finding derivations of lie algebras, Proyecciones Journal of Mathematics, 31(1): 8190.

[2] Dagli, Mehmet., 2004, Levi decomposition of Lie algebras; Algorithm for its computation, Master of Science Thesis, Iowa State University: 1218.

[3] Diatta, A., and Manga, B., 2014, On properties of principal elements of Frobenius Lie algebras, J. Lie Theory 24: 849 - 864.

[4] Hendrawan, R., 2020, Aljabar Simetrik Kiri Pada Aljabar Lie Frobenius Riil Berdimensi 6, Skripsi, tidak diterbitkan, Departemen Matematika FMIPA UNPAD

[5] Hilgert, J., dan Neeb, K.-H., 2012, Structure and Geometry of Lie Groups, New York: Springer Monographs in Mathematics, Springer 
[6] Humphreys, J., 1972, Introduction to Lie ALgebra and its Representation, New York Heidelberg Berlin: Springer-Verlag

[7] Ooms, A. I., 2009, Computing invariants and semi-invariants by means of Frobenius Lie algebras, J. Algebra 321 : 1293 - 1312

[8] Pham, D. N., 2016, G-Quasi-Frobenius Lie Algebras, Archivum Mathematicum, 52(4): $233-262$. 